\title{
Calculation of Arc-Circuit Asymmetry in Electric-Arc Furnaces
}

\section{Peter F. Ryff}

Ryerson University

digital.library.ryerson.ca/object/334

Please Cite:

Ryff, P. F. (1983). Calculation of arc-circuit asymmetry in electric-arc furnaces.

IEE Proceedings B - Electric Power Applications, 130(3), 213-217.

$\underline{\text { doi:10.1049/ip-b.1983.0032 }}$

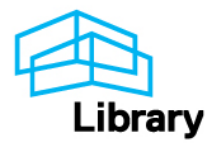




\title{
Calculation of arc-circuit asymmetry in electric-arc furnaces
}

\author{
Prof. P.F. Ryff, M.A.Sc., Ph.D., Sen. Mem. I.E.E.E., Member A.P.E.O. \\ Indexing terms: Arcing, Industrial applications of power

\begin{abstract}
A method is described to determine the arc voltages on a per-phase basis of a 3-phase direct electricarc furnace. Having determined the arc voltages, other arc parameters, such as arc resistance, length and power, can then be obtained. By using the procedure outlined, in conjunction with experimental data on a $100 \mathrm{t}, 5 \frac{1}{2} \mathrm{~m}$ diameter shell furnace having a triangulated secondary electrical system, it will be shown that controlling arc powers by means of the impedance control method still results in asymmetric furnace operation. Sample calculations are included, illustrating actual conditions for this furnace.
\end{abstract}

\section{Introduction}

The uneven arc-power levels at the tips of the electrodes during furnace operation is a well known fact, and they generally result in imbalanced circuit conditions. Ideally, the arc voltage and current should be measured and their product representing the arc power controlled by the electronic control system, in order to ensure even powers. However, direct measurement of the arc voltage cannot be carried out during operation, for obvious reasons, such as the furnace atmosphere and the fact that the system has a floating neutral. Therefore, the current and voltage is measured at the furnace transformer and the resulting impedance $Z=V / I$ taken as an operational value. Electrode control systems operating on this scheme are referred to as 'impedance controlled'. Arc powers are controlled by the total phase impedance, regardless of their phase angle. Provided the system is linear and the secondary circuit is ideally triangulated, the phases can be constructed such that they are electrically equal. The operation of an arc furnace, however, is more complicated. This is mainly because of two separate phenomena, one being the impedance of the furnace, the other attributable to the arc itself. The secondary circuit of a modern arc furnace is triangulated throughout. If no restrictions are placed by the meltshop, such as height requirements, equilateral triangulation is strived for. However, on the larger furnaces there are several parallel flexible cables, as well as parallel bus tubes on the electrode arms. Thus, having multiple conductors in parallel, per-phase various geometrical configurations are possible, none constituting ideal equilateral triangular systems. For instance, for the furnace under discussion there are four flexible cables and two bus tubes per phase, and the triangulation employed is as shown in Fig. 1. As can be seen, the distances between the cables, and the bus tubes are not all equal and, therefore, their mutual inductances vary. Consequently, the phase resistances and reactances are unequal, giving rise to asymmetry in the furnace, ultimately exhibiting itself in uneven side-wall wear of the furnace lining. Naturally efforts are made to minimise any resulting imbalance, but ideal conditions are not achieved. Furthermore, it must be realised that the resistances and reactances of the individual phases are not really constant. This is owing to unavoidable changes in circuit configuration caused by uneven charging levels under the electrodes, moving the electrode arms from the desired triangulated configuration. At the same time, the phase currents continually change, resulting in movement of the flexible cables in response to changing electromagnetic forces. Cables in the phases are

Paper 2347B(P4), first received 11 th May and in revised form 19th November 1982

The author is with the Ryerson Polytechnical Institute, Department of Electrical Engineering Technology, 50 Gould St., Toronto, Ont., Canada M5B IE8 pulled in a tighter configuration, while at the same time the cables between phases swing apart when, for instance, the current increases. These combined effects modify the reflected resistances and coupled reactances continuously, thereby in turn altering the phase impedances [1]. In addition, the electric arcs themselves introduce nonlinearities, especially during the initial period of a heat, since their voltage waveforms are rarely sinusoidal. Since the arc resistance depends upon the arc voltage, the impedance is affected even more.
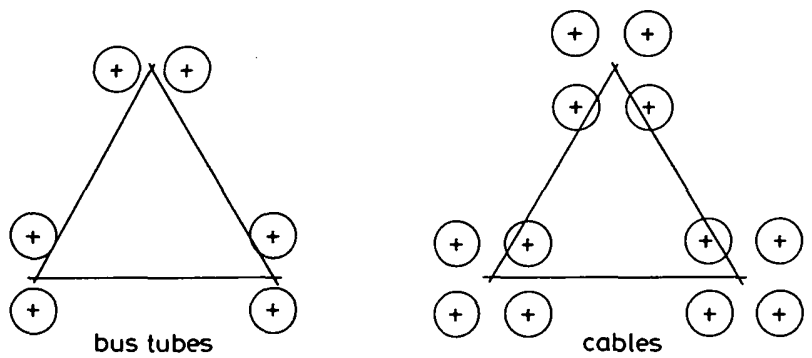

Fig. 1 Schematic of triangulation of furnace secondary electrical circuit

In this paper, a procedure is outlined to determine the arc voltages. Knowing these, all other arc-circuit parameters can then be determined. As a specific example, the results are applied to a furnace installation for which numerous experimental data are available. It will be shown that, in this case, representing a typical installation of good design, the imbalance, although modest, is present.

\section{Derivation of arc-vol tage equation}

The secondary electrical circuit of an electric-arc furnace from the transformer secondary tap on can be schematically represented by an electric circuit, as illustrated in Fig. 2. The phase impedances $Z_{i}=r_{i}+j x_{i}, i=1,2,3$ consist of the equivalent resistances and reactances per phase, which accounts for the mutual inductances and reflected resistances. This representation of the equivalent circuit is applicable for all furnaces, whether balanced or not. $V_{i}$ and $I_{i}, i=1,2,3$, denote the phase voltages and currents, respectively. Applying Kirchhoff's laws, the following relations can be stated:

$$
\begin{aligned}
& \bar{V}_{1}-\bar{V}_{2}=\bar{I}_{1} \bar{Z}_{1}-\bar{I}_{2} \bar{Z}_{2}+\bar{v}_{1}-\bar{v}_{2} \\
& \bar{V}_{2}-V_{3}=\bar{I}_{2} \bar{Z}_{2}-\bar{I}_{3} \bar{Z}_{3}+\bar{v}_{2}-\bar{v}_{3} \\
& \bar{I}_{1}+\bar{I}_{2}+\bar{I}_{3}=0
\end{aligned}
$$

where $v_{i}, i=1,2,3$, represents the arc-voltage drops across the arc resistances $R_{i}$, respectively, in the corresponding phases.

Considering the phase relationships between the various 
electrical parameters, as shown in Fig. 3, eqn. 1 can be written as

$$
\begin{aligned}
& V_{1}-V_{2}\left(\cos \phi_{2}+j \sin \phi_{2}\right)=I_{1}\left(\cos \alpha_{1}+j \sin \alpha_{1}\right) \times \\
& \left(r_{1}+j x_{1}\right)-I_{2}\left(\cos \alpha_{2}+j \sin \alpha_{2}\right)\left(r_{2}+j x_{2}\right) \\
& +v_{1}\left(\cos \alpha_{1}+j \sin \alpha_{1}\right)-v_{2}\left(\cos \alpha_{2}+j \sin \alpha_{2}\right)
\end{aligned}
$$

where $\phi_{i}$ and $\alpha_{i}, i=1,2,3$, are the angles associated with the various electrical quantities, as illustrated in Fig. 3. The arc voltage and current are assumed to be in phase. Eqns. 2 and 3 can be similarly treated. Separating the resulting equations into their real and imaginary components, realising that

$$
r_{i}=Z_{i} \cos \alpha_{z_{i}} \quad x_{i}=Z_{i} \sin \phi_{z_{i}}
$$

and making use of the trigonometric identity

$$
\cos \left(\alpha_{i}+\phi_{z_{i}}\right)=\cos \alpha_{i} \cos \phi_{z_{i}}-\sin \alpha_{i} \sin \phi_{z_{i}}
$$

there results six equations, namely:

$$
\begin{aligned}
& D_{4}+v_{2} \cos \alpha_{2}-v_{1} \cos \alpha_{1} \\
& \quad=D_{1} \cos \left(\alpha_{1}+\phi_{z_{1}}\right)-D_{2} \cos \left(\alpha_{2}+\phi_{z_{2}}\right) \\
& D_{5}+v_{2} \sin \alpha_{2}-v_{1} \sin \alpha_{1} \\
& \quad=D_{1} \sin \left(\alpha_{1}+\phi_{z_{1}}\right)-D_{2} \sin \left(\alpha_{2}+\phi_{z_{2}}\right) \\
& D_{6}+v_{3} \cos \alpha_{3}-v_{2} \cos \alpha_{2} \\
& \quad=D_{2} \cos \left(\alpha_{2}+\phi_{z_{2}}\right)-D_{3} \cos \left(\alpha_{3}+\phi_{z_{3}}\right) \\
& D_{7}+v_{3} \sin \alpha_{3}-v_{2} \sin \alpha_{2} \\
& \quad=D_{2} \sin \left(\alpha_{2}+\phi_{z_{2}}\right)-D_{3} \sin \left(\alpha_{3}+\phi_{z_{3}}\right) \\
& I_{1} \cos \alpha_{1}+I_{2} \cos \alpha_{2}+I_{3} \cos \alpha_{3}=0 \\
& I_{1} \sin \alpha_{1}+I_{2} \sin \alpha_{2}+I_{3} \sin \alpha_{3}=0
\end{aligned}
$$

where the following notation of symbols is used to facilitate presentation of the various expressions:

$$
\begin{aligned}
& D_{1}=I_{1} Z_{1} \\
& D_{2}=I_{2} Z_{2} \\
& D_{3}=I_{3} Z_{3} \\
& D_{4}=V_{1}-V_{2} \cos \phi_{2}
\end{aligned}
$$
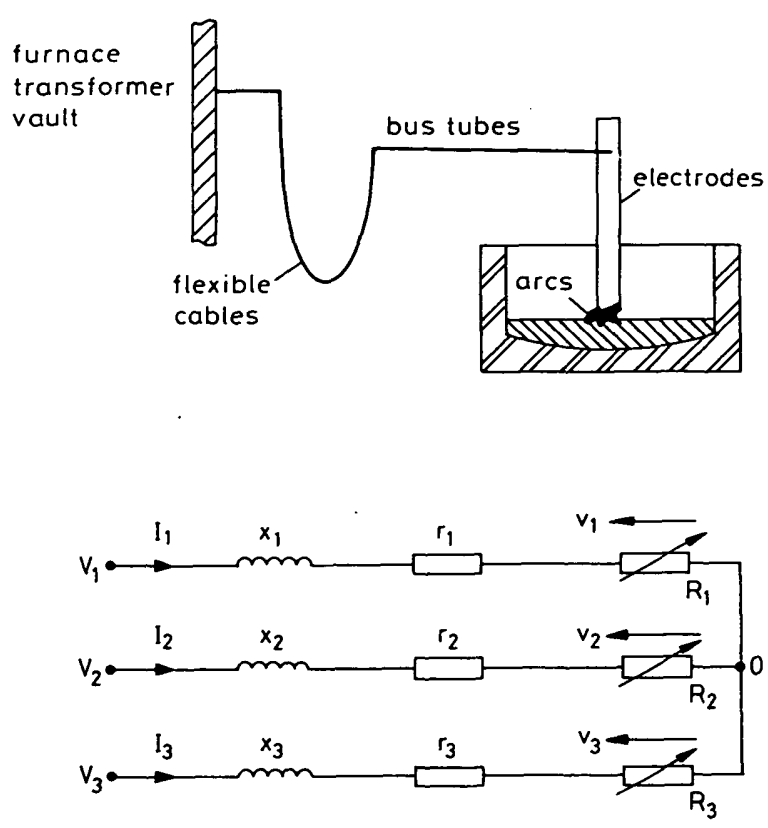

Fig. 2 3-phase electric-arc furnace and its circuit

$$
\begin{aligned}
& D_{5}=-V_{2} \cos \phi_{2} \\
& D_{6}=V_{2} \cos \phi_{2}-V_{3} \cos \phi_{3} \\
& D_{7}=V_{2} \sin \phi_{2}-V_{3} \sin \phi_{3}
\end{aligned}
$$

and $\phi_{z_{i}}, i=1,2,3$, are the phase angles associated with the phase impedances $Z_{i}$, exclusive of the arc resistances, which are obtained from a short-circuit test (dip test) [23]. The quantities $D_{1} \ldots D_{7}$ can be calculated from the measured input data; since the line-to-line voltages, as well as the phase currents, are subject to measurement, the angles $\phi_{i}$ are associated with the 3-phase voltage supply.

Thus, there are six equations, eqns. 4-9, which must be solved. From eqns. 4 and 5 , eliminating $v_{2}$ by multiplying eqn. 4 by $\sin \alpha_{2}$ and eqn. 5 by $\cos \alpha_{2}$, and subtracting the resulting equations from one another yields:

$$
\begin{gathered}
D_{5} \cos \alpha_{2}-D_{4} \sin \alpha_{2}-v_{1}\left(\sin \alpha_{1} \cos \alpha_{2}-\cos \alpha_{1} \sin \alpha_{2}\right) \\
=D_{1}\left\{\sin \left(\alpha_{1}+\phi_{z_{1}}\right) \cos \alpha_{2}-\cos \left(\alpha_{1}+\phi_{z_{1}}\right) \sin \alpha_{2}\right\} \\
-D_{2}\left\{\sin \left(\alpha_{2}+\phi_{z_{2}}\right) \cos \alpha_{2}-\cos \left(\alpha_{2}+\phi_{z_{2}}\right) \sin \alpha_{2}\right\}
\end{gathered}
$$

Using the trigonometric identity

$$
\sin (x-y)=\sin x \cos y-\cos x \sin y
$$

and letting $\alpha_{1}-\alpha_{2}=\alpha_{12}$, eqn. 11 gives the following expression when solved for the arc voltage in phase 1 , namely:

$$
\begin{aligned}
v_{1}= & \left\{D_{1} \sin \left(\alpha_{12}+\phi_{z_{1}}\right)-D_{2} \sin \phi_{z_{2}}+D_{4} \sin \alpha_{2}\right. \\
& \left.-D_{5} \cos \alpha_{2}\right\} /\left(-\sin \alpha_{12}\right)
\end{aligned}
$$

The arc voltage in phase 2 can similarly be obtained from eqns. 4 and 5 by eliminating $v_{1}$. To do so, multiply eqn. 4 by $\sin \alpha_{1}$ and eqn. 5 by $\cos \alpha_{2}$. This results after subtracting one equation from the other and solving for $v_{2}$, in

$$
\begin{aligned}
v_{2}= & \left\{D_{1} \sin \phi_{z_{1}}+D_{2} \sin \left(\alpha_{12}-\phi_{z_{2}}\right)+D_{4} \sin \alpha_{1}\right. \\
& \left.-D_{5} \cos \alpha_{1}\right\} /\left(-\sin \alpha_{12}\right)
\end{aligned}
$$

Finally, the third arc voltage in phase 3 is obtained from eqn. 6 by solving for $v_{3}$, resulting in

$$
\begin{aligned}
v_{3}= & \left\{D_{2} \cos \left(\alpha_{2}+\phi_{z_{2}}\right)-D_{3} \cos \left(\alpha_{3}+\phi_{z_{3}}\right)\right. \\
& \left.+v_{2} \cos \alpha_{2}-D_{6}\right\} / \cos \alpha_{3}
\end{aligned}
$$

At this point, eqns. 12 to 14 cannot be solved for the arc voltages, since the angles $\alpha_{i}$ are as yet unknown. Therefore,

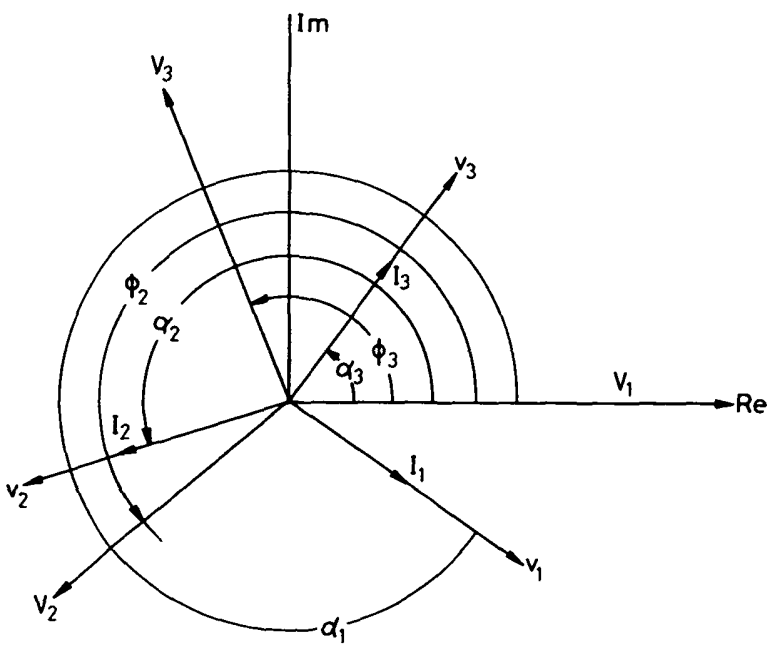

Fig. 3 Phasor diagram showing notation of variables used 
expressions for these parameters will now be derived. From eqns. 4 and 5 , the following relation is obtained by eliminating the $v_{1}$-term:

$$
\begin{aligned}
& D_{4} \sin \alpha_{1}-D_{5} \cos \alpha_{1}+v_{2} \sin \alpha_{12} \\
& \quad=-D_{1} \sin \phi_{z_{1}}-D_{2} \sin \left(\alpha_{12}-\phi_{z_{2}}\right)
\end{aligned}
$$

Similarly, from eqns. 6 and 7 , the $v_{3}$-term is eliminated, giving

$$
\begin{gathered}
D_{7} \cos \alpha_{3}-D_{6} \sin \alpha_{3}-v_{2} \sin \alpha_{23} \\
=D_{2} \sin \left(\alpha_{23}+\phi_{z_{2}}\right)-D_{3} \sin \phi_{z_{3}}
\end{gathered}
$$

where $\alpha_{23}=\alpha_{2}-\alpha_{3}$.

Before proceeding, it should be realised that

$$
\alpha_{23}=\cos ^{-1}\left(I_{1}^{2}-I_{2}^{2}-I_{3}^{2}\right) /\left(2 I_{2} I_{3}\right)
$$

which is the cosine law applied to the current triangle, since

$$
\bar{I}_{1}+\bar{I}_{2}+\bar{I}_{3}=0
$$

Likewise, by manipulating eqns. 8 and 9 , it is readily shown that

$$
\sin \alpha_{23} / \sin \alpha_{12}=I_{1} / I_{3}
$$

and

$$
\sin \alpha_{13} / \sin \alpha_{12}=-I_{2} / I_{3}
$$

Therefore, by multiplying eqn. 15 by $\sin \alpha_{23}$ and eqn. 16 by $\sin \alpha_{12}$, and subtracting the resulting equations from one another, the $v_{2}$-term will be eliminated. If further simplifications are made by substituting eqns. 18 and 19 there results

$$
\begin{aligned}
& D_{4} I_{1} \sin \alpha_{1} / I_{3}-D_{5} I_{1} \cos \alpha_{1} / I_{3}-D_{6} \sin \alpha_{3} \\
& \quad+D_{7} \cos \alpha_{3}=D_{8}
\end{aligned}
$$

where

$$
D_{8}=-\left\{D_{1} I_{1} \sin \phi_{z_{1}}+D_{2} I_{2} \sin \phi_{z_{2}}+D_{3} I_{3} \sin \phi_{z_{3}}\right\} / I_{3}
$$

Eqn. 20 can now be reduced to an equation having only $\alpha_{3}$ as an unknown, by letting $\alpha_{1}=\alpha_{13}+\alpha_{3}$ and $\alpha_{13}=\alpha_{12}+\alpha_{23}$. After substitution of these expressions and some trigonometric manipulation, eqn. 20 becomes:

$$
\begin{gathered}
-D_{4} D_{9} \cos \alpha_{3}+D_{4} D_{10} \sin \alpha_{3}-D_{5} D_{10} \cos \alpha_{3} \\
-D_{5} D_{9} \sin \alpha_{3}-D_{6} \sin \alpha_{3}+D_{7} \cos \alpha_{3}=D_{8}
\end{gathered}
$$

with

$$
D_{9}=-I_{2} \sin \alpha_{23} / I_{3}
$$

and

$$
D_{10}=I_{1} \cos \alpha_{13} / I_{3}
$$

Collecting terms and solving for $\cos \alpha_{3}$, gives

$$
\cos \alpha_{3}=\left(D_{8}-E_{1} \sin \alpha_{3}\right) / E_{2}
$$

where

$$
E_{1}=D_{4} D_{10}-D_{5} D_{9}-D_{6}
$$

and

$$
E_{2}=-D_{4} D_{9}-D_{5} D_{10}+D_{7}
$$

Although eqn. 25 expresses $\cos \alpha_{3}$ in terms of $\sin \alpha_{3}$, the angle $\alpha_{3}$ can be determined, realising

$$
\sin ^{2} \alpha_{3}=1-\cos ^{2} \alpha_{3}
$$

Substitution of eqn. 25 into eqn. 28 and solving the resulting quadratic equation in $\sin \alpha_{3}$, yields

$$
\sin \alpha_{3}=\left\{E_{1} D_{8} \pm E_{2}\left(E_{1}^{2}+E_{2}^{2}-D_{8}^{2}\right)^{1 / 2}\right\} /\left(E_{1}^{2}+E_{2}^{2}\right)
$$

Thus, all necessary parameters can be obtained in the solution for the arc voltages as given by eqns. 12 to 14 . The numerical example included will illustrate the sequence of calculations to determine the desired quantities.

\section{Measurement data on $100 \mathrm{t}$ EAF}

The electrical operating characteristics for the furnace at five different transformer tap positions are presented in Table 1, which also gives the data taken during a 3-phase short-circuit test (from the so-called dip test) performed at tap no. 8 .

These electrical measurements were taken by two methods. One method employed watt hour, VAr hour and $\mathrm{V}^{2}$ hour measurements against time in several increments by pulsing instruments connected at the furnace transformer primary metering point for the commonly used tap positions 33

\begin{tabular}{|c|c|c|c|c|c|c|c|c|c|}
\hline \multirow{2}{*}{$\begin{array}{l}\text { Tap } \\
\text { position }\end{array}$} & \multirow{2}{*}{$\begin{array}{l}\text { line/line } \\
\text { voltage } \\
\text { (loaded) }\end{array}$} & \multirow[t]{2}{*}{ MW } & \multirow[t]{2}{*}{ MVAr } & \multirow[t]{2}{*}{ MVA } & \multirow[t]{2}{*}{ PF } & \multirow{2}{*}{$\begin{array}{l}\text { average } \\
\text { operating } \\
\text { reactance }\end{array}$} & \multicolumn{3}{|c|}{ Secondary currents, kA } \\
\hline & & & & & & & $\begin{array}{l}\text { Floor } \\
I_{1}\end{array}$ & $\begin{array}{l}\text { Centre } \\
I_{2}\end{array}$ & $\begin{array}{l}\text { Pit } \\
I_{3}\end{array}$ \\
\hline 33 & 539 & 39.96 & 40.13 & 56.63 & 0.706 & 3.64 & 61.3 & 62.0 & 58.6 \\
\hline 29 & 509 & 36.25 & 39.43 & 53.56 & 0.677 & 3.56 & 61.9 & 61.3 & 59.1 \\
\hline 25 & 478 & 32.07 & 38.23 & 49.90 & 0.643 & 3.52 & 61.5 & 60.3 & 58.7 \\
\hline 20 & 444 & 27.38 & 35.81 & 45.08 & 0.607 & 3.46 & 58.9 & 59.1 & 58.2 \\
\hline 10 & 372 & 19.55 & 26.82 & 33.19 & 0.589 & 3.36 & 51.8 & 51.1 & 51.8 \\
\hline $8^{*}$ & 348 & 3.69 & 38.94 & 39.11 & 0.094 & 3.085 & 64.6 & 72.3 & 61.0 \\
\hline
\end{tabular}
through 10 . The other measurements, taken concurrently with these measurements, were monitored by means of averaging meters $\left(I^{2} d t\right)$ in the primary-current transformer circuit.

From the acquired data of the dip test, the furnace impedance, both resistive and reactive components, on a perphase basis, can be calculated, using either of the methods outlined elsewhere $[2,3]$.

From the measurement data, the arc characteristics will now be determined, such as arc power, arc length, arc resistance and arc voltage. This will be done in the following example for tap position 33, using the method described. Results for the remaining tap positions are listed in Table 2.

\subsection{Sample calculation}

Using the method outlined, the following sequence of calculations gives the results for the arc voltages in their respective phases. The data for tap no. 33 are used (Table 1). The supply voltages on a per-phase basis are obtained from the measured primary line to line voltages and, taking $V_{1}$ as the reference

Table 1: Measured furnace operating data obtained from the primary metering point

${ }^{\text {* }}$ Results for short-circuit test (no harmonics present in system and arc resistances equal to zero) 
Table 2: Calculated operational values

\begin{tabular}{|c|c|c|c|c|c|c|c|c|}
\hline \multirow[t]{2}{*}{ Tap no. } & \multirow{2}{*}{$\begin{array}{l}V_{p h} \\
V\end{array}$} & \multirow{2}{*}{$\begin{array}{l}\text { Harmonic content } \\
\%\end{array}$} & \multicolumn{3}{|c|}{ Phase reactance, $\mathrm{m} \Omega$} & \multicolumn{3}{|c|}{ Phase resistance, $\mathrm{m} \Omega$} \\
\hline & & & 1 & 2 & 3 & 1 & 2 & 3 \\
\hline 33 & 311 & 17 & 3.70 & 3.04 & 4.10 & 3.17 & 3.58 & 4.12 \\
\hline 29 & 294 & 15 & 3.63 & 2.98 & 4.03 & 2.73 & 3.31 & 3.87 \\
\hline 25 & 276 & 14 & 3.60 & 2.96 & 3.99 & 2.36 & 3.00 & 3.56 \\
\hline 20 & 256 & 12 & 3.54 & 2.91 & 3.92 & 2.12 & 2.81 & 3.06 \\
\hline 10 & 215 & 8.9 & 3.44 & 2.82 & 3.81 & 1.79 & 2.72 & 2.81 \\
\hline \multicolumn{3}{|c|}{ Phase arc voltage, $\mathrm{V}$} & \multicolumn{3}{|c|}{ Phase impedance $\mathrm{m} \Omega$} & \multicolumn{3}{|c|}{ Phase angle, degrees } \\
\hline 1 & 2 & 3 & 1 & 2 & 3 & 1 & 2 & 3 \\
\hline 176.1 & 208.8 & 217.0 & 4.87 & 4.70 & 5.81 & 49.4 & 40.3 & 44.9 \\
\hline 150.6 & 189.9 & 204.3 & 4.54 & 4.45 & 5.57 & 53.1 & 42.0 & 46.2 \\
\hline 126.4 & 168.3 & 185.1 & 4.30 & 4.21 & 5.35 & 56.8 & 44.6 & 48.3 \\
\hline 107.3 & 153.5 & 154.1 & 4.12 & 4.05 & 4.97 & 59.1 & 46.0 & 52.0 \\
\hline 77.2 & 128.3 & 124.4 & 3.88 & 3.92 & 4.73 & 62.5 & 46.0 & 53.6 \\
\hline \multicolumn{3}{|c|}{ Phase arc power, MW } & \multicolumn{3}{|c|}{ Phase resistance, $\mathrm{m} \Omega$} & \multicolumn{3}{|c|}{ Phase arc length, $\mathrm{cm}$} \\
\hline 1 & 2 & 3 & 1 & 2 & 3 & 1 & 2 & 3 \\
\hline 10.79 & 12.95 & 12.71 & 2.87 & 3.37 & 3.71 & 12.2 & 14.9 & 15.5 \\
\hline 9.31 & 11.64 & 12.08 & 2.43 & 3.10 & 3.46 & 10.0 & 13.3 & 14.5 \\
\hline 7.80 & 10.10 & 10.90 & 2.06 & 2.79 & 3.15 & 8.0 & 11.6 & 13.0 \\
\hline 6.32 & 9.07 & 8.97 & 1.82 & 2.60 & 2.65 & 6.4 & 10.3 & 10.3 \\
\hline 4.0 & 6.56 & 6.44 & 1.49 & 2.51 & 2.40 & 3.9 & 8.2 & 7.9 \\
\hline \multicolumn{3}{|c|}{ Phase currents, kA } & \multicolumn{3}{|c|}{$\%$ phase current unbalance } & \multicolumn{3}{|c|}{$\%$ phase arc power unbalance } \\
\hline 1 & 2 & 3 & 1 & 2 & 3 & 1 & 2 & 3 \\
\hline 61.3 & 62.0 & 58.6 & 1.10 & 2.25 & -3.35 & -11.19 & 6.58 & 4.61 \\
\hline 61.9 & 61.3 & 59.1 & 1.87 & 0.88 & -2.74 & -15.44 & 5.72 & 9.72 \\
\hline 61.5 & 60.3 & 58.7 & 2.22 & 0.22 & -2.44 & -18.75 & 5.21 & 13.54 \\
\hline 58.9 & 59.1 & 58.2 & 0.28 & 0.62 & -0.91 & -22.17 & 11.70 & 10.47 \\
\hline 51.8 & 51.1 & 51.8 & 0.45 & -0.90 & 0.45 & -29.41 & 15.76 & 13.65 \\
\hline
\end{tabular}

phasor, there results for the per-phase voltages:

$$
\begin{aligned}
& V_{1}=539 / \sqrt{3} \angle 0^{\circ}=311 \angle 0^{\circ} \quad V_{2}=311 \angle-120^{\circ} \\
& V_{3}=311 \angle 120^{\circ}
\end{aligned}
$$

It should be noted that the impedance of the furnace transformer in the overall furnace circuit is a negligible component. Therefore, any additional unbalances which may occur owing to transformer unbalance may be neglected and the transformed voltages assumed to be a balanced set on the secondary side.

From the short-circuit test data, the average secondary reactance is $3.085 \mathrm{~m} \Omega$, and the individual phase reactances determined by the methods referred to earlier $[2,3]$ are: $x_{1}=$ $3.158 \mathrm{~m} \Omega, x_{2}=2.594 \mathrm{~m} \Omega$ and $x_{3}=3.502 \mathrm{~m} \Omega$. The average secondary reactance during operation was measured to be $3.640 \mathrm{~m} \Omega$, as indicated in Table 1 and calculated from the data obtained during the test, as described in Section 3.

The unbalance in reactances is evident and primarily results from the unavoidable fact that the three phases cannot be constructed electrically identical. Although the outside phases can be similar, the centre arm in most designs is shorter. Furthermore, when the furnace is operating, triangulation cannot be maintained throughout the secondary system, because of, for instance, uneven electrode wear and uneven charging levels under the electrodes. This results in mutual inductance effects between phases which complicates matters.

In addition, the average secondary reactance during operation is $17 \%$ higher than that obtained during the short-circuit test. Many authors attribute this increase to the harmonic content introduced into the circuit by nonsinusoidal arcvoltage waveforms. In part, this may be true, since the arc can demonstrate preferential conduction in a form of rectification [4]. Also, the arc plasma exhibits thermal hysteresis which manifests itself by creating even harmonics. A fact that has not been considered so far is the influence of the nonlinear behaviour of the magnetic material surrounding the electrical system. All these matters combined should explain this apparent increase in the operational reactance. To attribute the reactance increase solely to arc behaviour appears mislead. ing at best.

Therefore, the reactances obtained from the short-circuit test are adjusted accordingly, to represent a more accurate operational value.

The phase resistances are assumed to be unaffected. Thus:

$$
\begin{aligned}
z_{1} & =0.299+j 1.17 \times 3.158=0.299+j 3.695 \\
& =3.707 \not 85.4^{\circ} \mathrm{m} \Omega
\end{aligned}
$$

similarly,

$$
z_{2}=0.213+j 3.035=3.042 \angle 86.0^{\circ} \mathrm{m} \Omega
$$

and

$$
z_{3}=0.411+j 4.097=4.118 \not 84.3 \mathrm{~m} \Omega
$$

Knowing these parameters enables calculation of the values $D_{1}$ to $D_{7}$, according to eqns. $10 a-g$, namely:

$$
\begin{aligned}
& D_{1}=61300 \times 3.707 \times 10^{-3}=227.2 \\
& D_{2}=188.6 \quad D_{3}=241.2 \\
& D_{4}=311=311 \cos \left(-120^{\circ}\right)=466.5 \\
& D_{5}=269.3 \quad D_{6}=0 \quad D_{7}=-538.6
\end{aligned}
$$

From eqn. 17,

$$
\begin{aligned}
& \qquad \begin{aligned}
\alpha_{23}= & \cos ^{-1}\left(61.3^{2}-62.0^{2}-58.6^{2}\right) /(2 \times 62 \times \\
\text { Likewise } & 58.6)=119^{\circ} \\
\alpha_{12}= & \cos ^{-1}\left(58.6^{2}-61.3^{2}-62.0^{2}\right) /(2 \times 61.3 \times 62.0) \\
= & 123^{\circ}
\end{aligned}
\end{aligned}
$$

Therefore

$$
\begin{aligned}
\alpha_{13} & =\alpha_{1}-\alpha_{2}+\alpha_{2}-\alpha_{3}=\alpha_{12}+\alpha_{23}=123+119 \\
& =242^{\circ}
\end{aligned}
$$


It is now possible to determine the quantities $D_{8}$ to $D_{10}$. From eqns. 21,23 and 24 :

$$
\begin{aligned}
D_{8}= & -\left[227.2 \times 61.3 \times \sin 85.4^{\circ}+188.6 \times 62.0 \times\right. \\
& \left.\sin 86.0^{\circ}+241.2 \times 58.6 \times \sin 84.3^{\circ}\right]=-676.2 \\
D_{9}= & \frac{69.29}{58.56} \times \sin 119^{\circ}=0.926 \\
D_{10}= & \frac{61.29}{58.56} \times \cos 242^{\circ}=-0.491
\end{aligned}
$$

Therefore, the values of $E_{1}$ and $E_{2}$, according to eqns. 26 and 27 , can be obtained, giving

$$
\begin{aligned}
E_{1} & =466.5 \times-0.491-269.3 \times 0.926-0=-478.4 \\
E_{2} & =-466.5 \times 0.926+269.3 \times 0.491-538.6 \\
& =-838.4
\end{aligned}
$$

which are needed to solve for $\sin \alpha_{3}$ as determined by eqn. 29 , resulting in $\sin \alpha_{3}=0.967$ and $\alpha_{3}=75.2^{\circ}$.

Although the solution to the quadratic equation gives two possible answers, the one that fits the physical situation must be chosen, which determines the sign in front of the root sign as being positive. Knowing $\alpha_{3}$, the remaining calculations are straightforward, leading to $\alpha_{2}=\alpha_{3}+\alpha_{23}=75+119=194^{\circ}$ and $\alpha_{1}=\alpha_{2}+\alpha_{12}=194+123=317^{\circ}$, giving all the necessary values for the computation of the arc voltages according to eqns. $12-14$, yielding

$$
v_{1}=176.1 \mathrm{~V} \quad v_{2}=208.8 \mathrm{~V} \quad v_{3}=217.0 \mathrm{~V}
$$

Having determined these, the arc powers $P_{\text {arc }}=I_{i} \times v_{i}, i=1$, 2,3 , as well as the arc resistances $R_{i}=v_{i} / I_{i}$, ohmic losses in the secondary phases, total power etc. are easily found.

The remaining quantity of interest, the arc length $l_{i}$, can be determined by using established research results on electric arcs. It has been determined that, at the anode and cathode interfaces, a voltage drop of $30 \mathrm{~V}$ exists, and, along the arc length, a potential drop of $12 \mathrm{~V} / \mathrm{cm}$ occurs; although recent published data give somewhat different values $[5,6]$.

From this,

$$
v_{\text {arc }}=30+12 l_{\text {arc }} \mathrm{V}
$$

and the arc powers

$$
P_{\text {arc }}=v_{\text {arc }} \times I_{\text {arc }}
$$

Therefore, on substitution of $v_{\text {arc }}$ into the expression for arc power and solving for the arc length, this gives

$$
l_{\text {arc }}=\frac{P_{\text {arc }}}{12 I_{\text {arc }}}-2.5 \mathrm{~cm}
$$

These values of arc length associated with the voltages are also calculated in each instance and all results are tabulated in Table 2 for each voltage tap for comparison.

From the tabulated results, it can be seen that the controlled quantity, namely the phase impedance, is fairly even on a per-phase basis. The input voltages from the furnace transformer secondary are balanced and the secondary currents are about equal. Even so, symmetry of the arcs is not guaranteed.

The existing unbalance in arc powers is not only considerable but undesirable. To balance out the power levels (in this specific case), it is necessary to decrease the arc length under electrode no. 1 even further and/or lengthen the arc lengths for the remaining two electrodes.

An alternative method to increase the power for electrode no. 1 to the required level is to shorten the arc length in the phase which electrically follows the phase in which the higher power is desired [7]; i.e. the arc length for electrode no. 2 can be shortened for a phase sequence 123 . In any event, attempting to balance the powers for the electrodes has the sub. sequent result of unbalancing the electrode currents. This implies unbalanced operation conditions and uneven network loading, which may even be more undesirable.

\section{Conclusions}

The method presented makes it possible to determine the arc voltages and to obtain further information regarding the arccircuit parameters in their respective phases. It has also been shown that to maintain equal impedance magnitudes in all phases does not necessarily imply identical phase angles, and hence arc powers. Although the phase currents are slightly unbalanced, together with highly unequal arc-circuit parameters, the arc powers are greatly unbalanced - a condition normally experienced in practice. Efforts to equalise the arc powers are generally at the expense of a current unbalance, which, in many ways, is just as undesirable from the viewpoint of network loading.

However, it is the equalisation of the arc powers at the electrode tips which is of prime concern. The need for this is well documented in the literature - namely, to ensure even wear of the refractory lining.

It is expected that, to improve power equalisation more, and thereby accepting a moderate current unbalance, the metering point must be moved from the transformer side to the electrode arms. In so doing, the large variations in electrical parameters owing to cable movements are eliminated, ensuring that more reliable information is fed to the automatic electrodecontroller system.

\section{Acknowledgment}

The author wishes to thank the Union Carbide Co. for permission to publish their test results. Also special thanks are due to V. Bulat of EMPCO (Canada) Ltd., for valuable suggestions.

\section{References}

1 HOROSZKO, E.: Some remarks about the triangular arc furnace secondary conductor system. International Union for Electro Heat (UIE), 9-th International Congress, Cannes, October 1980

2 RYFF, P.F. and BULAT, V.: 'A new procedure to determine the EAF impedance'. Electrowarme International, Oct. 1981, pp. B250-B254

3 RYFF, P.F. and BULAT, V.: 'Determining the electric arc furnace circuit impedance from a single dip-test'. IEEE-IAS annual meeting conference record, 1981, pp. 952-956

4 BOWMAN, B.: 'Electrical characteristics of arc furnaces allowing for current swings'. Paper presented at International Union for Electro Heat (UIE) 8th international congress, Liege, 1976

5 FABER, H., and TIMM, K.: 'Ursache periodscher Spannungsschwankungen in Lichtbogeofen', Stahl u. Eisen, 1982, 102, pp. 159-164

6 BOWMAN, B., JORDAN, G.R., and FITZGERALD, F.: 'The physics of high-current arcs', J. Iron \& Steel Inst., June 1969, pp. 798-805

7 BAKER, G.M.: 'Electrical considerations in arc furnace productivity', AIME Electric Furnace Proceedings, 1979, pp. 333-337 